\title{
Ultra-long Cycle Life, Low-Cost Room Temperature Sodium-Sulfur Batteries Enabled by Highly Doped
}

\section{$(\mathrm{N}, \mathrm{S})$ Nanoporous Carbons}

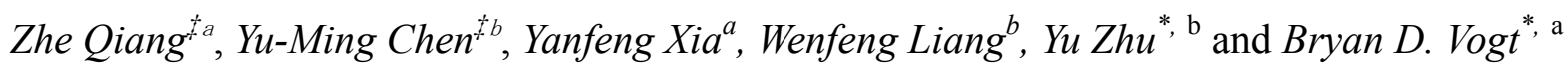

${ }^{\mathrm{a}}$ Department of Polymer Engineering, The University of Akron, 250 S Forge St, Akron, OH, 44325, United States

${ }^{b}$ Department of Polymer Science, Goodyear Polymer Center, The University of Akron, 170 University Circle, Akron, OH, 44325, United States

KEYWORDS. Na/S battery; Battery lifetime; Cycle life; carbonate-polysulfide reaction

ABSTRACT. Efficiency, cost, and lifetime are the primary challenges for stationary energy storage with vanadium-redox flow and sodium-sulfur batteries as promising options. In particular, room temperature sodium-sulfur battery systems offer the potential for safe, simple, low-cost and high energy density storage, but the high reactivity or solubility of sodium polysulfides in common liquid electrolytes for carbonates or glycols, respectively, leads to rapid performance loss on cycling. Herein, we demonstrate a robust route to mostly inhibit reactivity of the sulfides with carbonate electrolytes (and also inhibit the diffusion of polysulfides 
dissolved in TEGDME) and prevent performance loss on cycling using highly doped $(\approx 40$ atom\%) nanoporous carbon from low-cost raw materials infused with sulfur as the cathode. This cathode design leads to an ultra-stable room temperature sodium-sulfur battery with less than 3\% decay in the discharge capacity after 8,000 cycles at a high current density of $4.6 \mathrm{~A} / \mathrm{g}$. At 0.23 $\mathrm{A} / \mathrm{g}$, the discharge capacity is approximately $400 \mathrm{mAh} / \mathrm{g}$ and stable over 350 cycles. This combination of low cost and excellent cycle stability is promising for stationary, grid-level energy storage.

\section{Introduction}

Electrochemical energy storage (EES) has received intense attention for consumer electronics, grid and transportation applications. Efficient EES for grid storage is particularly important when combined with green but intermittent power sources, such as wind and solar [1, 2]. General considerations for performance of EES devices include rate capacity, charge-discharge efficiency, cycle life, cost and scalability [3]. For grid storage, the molten sodium-sulfur (Na-S) battery holds many advantages including the high natural abundance of sulfur and sodium for low-cost and higher energy density (theoretical specific energy density of $760 \mathrm{~W} \mathrm{~h} / \mathrm{kg}$ ) when compared to vanadium redox flow and lead-acid batteries [4, 5]. However, the requirement of high temperature operation $\left(300{ }^{\circ} \mathrm{C}-350{ }^{\circ} \mathrm{C}\right)$ can lead to explosions or corrosion that limits the battery safety [6]. This drawback has sparked interests in developing liquid electrolytes or solid polymer electrolyte membranes to provide satisfactory $\mathrm{Na}^{+}$conductivity at ambient temperature for $\mathrm{Na}-\mathrm{S}$ batteries and to further push the development of advanced Na-ion batteries [7-15]. However, the performance of room temperature Na-S batteries is generally limited by the poor electrical conductivity of sulfur, large volumetric variation $(\approx 170 \%)$, and high reactivity or solubility of 
polysulfides in most common liquid electrolytes that leads to poor cycle stability [12]. In order to address these problems, various sulfur cathode designs and separator modifications have been proposed [16 -23], which have been successful to address the first two issues, but the long term cycle stability of room temperature Na-S batteries is still challenging; recent designs enabled battery operation for over 1000 cycles, but the capacity fade was still significant $[24,25]$. For lithium-sulfur (Li-S) batteries, one of the general strategies for improving cycle stability through minimizing polysulfides shuttling is to introduce strong interactions of the sulfur molecules with heteroatom-doped carbons [26-28]. In these cases, the concentration of heteroatoms is generally limited $(<15$ at $\%)$ by the efficiency of carbon doping.

One critical challenge for room temperature metal-S batteries is the stability of the electrolyte [12], where instability can lead to significant capacity fade. Carbonate-based electrolytes have been shown to react irreversibly with lithium (poly)sulfides to generate electrochemically inactive chemical products $[18,29]$. This reactivity has led to the use of alternative solvent systems such as tetra(ethylene glycol) dimethyl ether (TEGDME) [30]. TEGDME is stable in the presence of sodium sulfides, but the polysulfides are highly soluble in TEGDME and thus it is critical to suppress polysulfide shuttling [30]. Interestingly, the infusion of $\mathrm{S}$ into polyacrylonitrile (PAN) prior to carbonization led to an electrode that provided fairly reversible performance for a room temperature Na-S battery when using EC:DEC as the electrolyte [18], which is counter to most reports for the use of carbonate based electrolytes. These PAN-S composites can also be used effectively in Li-S batteries to enable the use of carbonate electrolytes [31]. The carbonization of the PAN leads to N-doped carbon, so the interaction between $\mathrm{S}$ and N-doped carbons that decrease polysulfide shuttling [26-28] may also inhibit the 
reactions between $\mathrm{S}$ and carbonate-based electrodes. If this is the case, further improvements in the cycle stability of Na-S batteries using carbonate-based electrolytes may be achieved if the concentration of heteroatoms, in particular N, can be significantly increased (>15 at \%).

In order to address this challenge and determine if a higher heteroatom concentration will prevent significant capacity fade, we demonstrate a facile method to synthesize hierarchical porous carbon with high doping concentration $(\approx 40$ atom $\%)$ of nitrogen and sulfur from lowcost raw materials. The large heteroatom concentration in the carbon framework acts to effectively trap the sodium polysulfides in the nanopores through electrostatic interaction between the pore wall and sulfur and sodium-nitride $(\mathrm{Na}-\mathrm{N})$ bonds, enabling near complete inhibition of side reactions between polysulfides and the carbonate electrolyte. The inhibition of the reactivity with the carbonate electrolyte allows a significant improvement in the cycle stability of room temperature Na-S battery with an order of magnitude increase in the number of charge-discharge cycles $(>10000$ cycles at high rate of $4.6 \mathrm{~A} / \mathrm{g}$ in a CR2032 coin cell) demonstrated than previous reports for room temperature Na-S battery. The combination of low cost and ultra-long cycle life performance is promising for potential stationary battery applications. Additionally, we demonstrate inhibition of polysulfide diffusion in TEGDME through these highly doped nanoporous carbons, which suggests that these materials may also be used with other electrolyte systems to reduce polysulfide shuttling.

\section{Experimental Section}

\subsection{Synthesis of OMC and N,S-HPC:}


In a typical synthesis, $9.6 \mathrm{~g}$ Pluronic F127 (Sigma Aldrich) was initially dissolved in $8 \mathrm{~g}$ of $0.2 \mathrm{M}$ hydrochloride acid ( $\mathrm{HCl}$, Sigma Aldrich) and $24 \mathrm{~g}$ of ethanol (Sigma Aldrich) under $45^{\circ} \mathrm{C}$ for 2 h. Then, $12 \mathrm{~g}$ of resol solution (50 wt \% in ethanol, low molecular phenolic resin, synthesized according to previous report), $15 \mathrm{~g}$ tetraethyl orthosilicate (TEOS, Sigma Aldrich) were added into the solution. After stirring for $2 \mathrm{~h}$ at $40^{\circ} \mathrm{C}$, the solution was subsequently cast on the polyethylene terephthalate (PET) substrate $(125 \mu \mathrm{m}$, Terphane Inc) by doctor-blade casting at 50 $\mathrm{cm} / \mathrm{min}$. The film was heated at $50{ }^{\circ} \mathrm{C}$ for $3 \mathrm{~h}$ and then heated at $100^{\circ} \mathrm{C}$ for $3 \mathrm{~h}$ in order to remove the residual solvent and crosslink the resol. The crosslinked film was peeled from PET substrate and grinded into powder. For the synthesis of ordered mesoporous carbon (OMC), the powder was carbonized in a tubular furnace (SentroTech Inc.) under $\mathrm{N}_{2}$ atmosphere at $700{ }^{\circ} \mathrm{C}$ for 90 min. For synthesis of nitrogen, sulfur-doped hierarchical porous carbon (N,S-HPC), the powder was calcined in a tubular furnace (SentroTech Inc.) under $\mathrm{N}_{2}$ atmosphere at $350{ }^{\circ} \mathrm{C}$ for 90 min for removing the surfactants. Then, the powder was mixed and grinded with melamine (Sigma Aldrich) and benzyl disulfide (Sigma Aldrich) at a mass ratio of 1:25:5. The powder was carbonized in the tubular furnace under $\mathrm{N}_{2}$ atmosphere at $700{ }^{\circ} \mathrm{C}$ for $3 \mathrm{~h}$. The silica within the OMC and N,S-HPC was subsequently etched with daily refreshed $3 \mathrm{M} \mathrm{KOH}$ in ethanol : deionized water $(1: 1 ; \mathrm{v}: \mathrm{v})$ for 4 days. The powder was washed with deionized water and then dried under vacuum oven at $80^{\circ} \mathrm{C}$ for $24 \mathrm{~h}$.

\subsection{Synthesis of OMC/S and N,S-HPC/S Composites Cathodes and Coin Cell Fabrication:}

The OMC/S and N,S-HPC/S composites cathodes were fabricated following a typical meltdiffusion strategy. The carbon materials (OMC and N,S-HPC) were firstly mixed with sublimed sulfur powder with a mass ratio of 1:2 (C:S) by ball milling. Subsequently, the mixture was placed in a sealed vial at $155^{\circ} \mathrm{C}$ for $12 \mathrm{~h}$ in order to infiltrate sulfur into the pores. The 
sulfur/carbon composite was afterwards placed under a vacuum oven with an elevated temperature of $200^{\circ} \mathrm{C}$ to remove excess $\mathrm{S}$ on the surface of the carbon material.

The as prepared porous carbon/S composites were mixed with carbon black and styrene butadiene rubber: sodium salt of carboxymethyl cellulose (SBR:CMC) at a weight ratio of 70:20:10 by mortar and pestle to form a water based slurry. The slurry was then casted on the aluminum foil using a doctor blade prior to drying in an oven at $60^{\circ} \mathrm{C}$ overnight. The aluminum with slurry coating foil was punched into circular electrodes with the size of 5/16 in with an area mass loading of 1.0 1.1 mg/cm. Coin cells (CR2032, MTI Corp., USA) were assembled using sodium ingot as the counter electrode and $1 \mathrm{M}$ of sodium perchlorate $\left(\mathrm{NaClO}_{4}\right.$, Sigma Aldrich, $>98 \%$ ) in ethylene carbonate/propylene carbonate (EC/PC, 1:1 v/v\%) as the electrolyte [32]. Glass fiber $\left(\mathrm{GF} / \mathrm{B}\right.$, Whatman $\left.{ }^{\circledR}, \mathrm{USA}\right)$ was used as the separator.

\subsection{Characterizations:}

Scanning electron microscope (SEM) images and energy dispersive X-ray mapping (EDX mapping) were acquired by using JEOL-7401 field-emission scanning electron microscope. High-resolution transmission electron microscope (HRTEM) images and EDX mapping were obtained using Tecnai G2 F20 equipped with EDAX SUTW (super ultrathin window) from FEI with an accelerating voltage of $200 \mathrm{kV} . \mathrm{N}_{2}$ adsorption-desorption isotherms were measured using

a Micromeritics Tristar. The specific surface area was determined using Brunauer Emmett and Teller (BET) method and the pore size distributions were calculated from the adsorption isotherms using the Barrett, Joyner and Halenda (BJH) model. Transmission small angle X-ray scattering (SAXS) was performed on a Rigaku MicroMax 002+ instrument with $0.154 \mathrm{~nm}$ wavelength X-rays. X-ray diffraction (XRD) patterns were collected on a Rigaku instrument 
equipped with an $18 \mathrm{~kW}$ rotating anode X-ray generator in the transmission mode. The X-ray photoelectron spectroscopy (XPS) was performed on PHI5000 Versa Probe II Scanning XPS Microprobe (ULVAC-PHI, Inc.), for both survey and high-solution spectra associated with C1s, N1s and S2p using a $0.05 \mathrm{eV}$ step size and a pass energy of $11.75 \mathrm{eV}$. The scans were recorded at a takeoff angle of $45^{\circ}$. The quantitative analysis was performed with Igor Pro (Wavemetrics) after Shirley background subtraction. Detailed characters of various chemical bonds in each sample were characterized by Fourier transform infrared spectroscopy (Thermo Scientific iS50 FT-IR) by preparing $\mathrm{KBr}$ pellets. Electrochemical performance was elucidated using an 8 Channel Battery Analyzer (BST-8A, 1mA, MTI Corp., USA) at room temperature. For understanding the effect of temperature on the battery performance, the cell was kept in an isothermal chamber during cycling. Specific capacity is calculated based on the weight of active sulfur within the composite material. Cyclic voltammetry (CV) and electrochemical impedance spectroscopy (EIS) were conducted using CH Instruments workstation (CHI608E). The CV was performed at a scan rate of $0.5 \mathrm{mV} / \mathrm{s}$ from the range $0.8-2.7 \mathrm{~V}$. EIS measurements were taken within the frequency range of $100 \mathrm{kHz}$ to $0.1 \mathrm{~Hz}$ with a $5 \mathrm{mV}$ AC voltage amplitude. H-cell testing was conducted by coating the OMC material or N,S-HPC with PVDF binder at a ratio of 8:2 on a Celgard separator using a doctor blade. Sodium polysulfide was fabricated mixing $7 \mathrm{~mol}$ of $\mathrm{S}$ with $1 \mathrm{~mol}$ of $\mathrm{Na}_{2} \mathrm{~S}$ in tetraethylene glycol dimethyl ether (TEGDME, Sigma Aldrcich) solvent due to much higher solubility of $\mathrm{Na}_{2} \mathrm{~S}_{8}$ in TEGDEM than in carbonate-based electrolytes; the polysulfides were further diluted and mixed until no solid content could be observed. The polysulfides solution in TEGDME was injected into the left hand side of the H-cell; pure TEGDME was injected into the right hand side of the H-cell. Images were then taken at different selected time intervals to observe the migration of the polysulfides across the membrane. 


\section{Results and Discussion}

The nitrogen, sulfur-doped hierarchical porous carbon (N,S-HPC) was prepared via a customized roll-to-roll line and the synthesis strategy includes four steps as illustrated in Figure 1: (1) formation and solidification of ordered mesophases driven by triconsitutent self-assembly of organic-inorganic composites [33]; (2) calcination to remove the surfactants (F127); (3) reaction of the mesoporous resol with dopants (melamine and benzyl disulfide) and conversion to doped carbon and (4) removal of silica nanoparticles to introduce micropores. This method enables the scalable fabrication of this N,S-HPC to generate single gram to more than hundred-grams in a single batch depending on the desired quantity [34, 35].

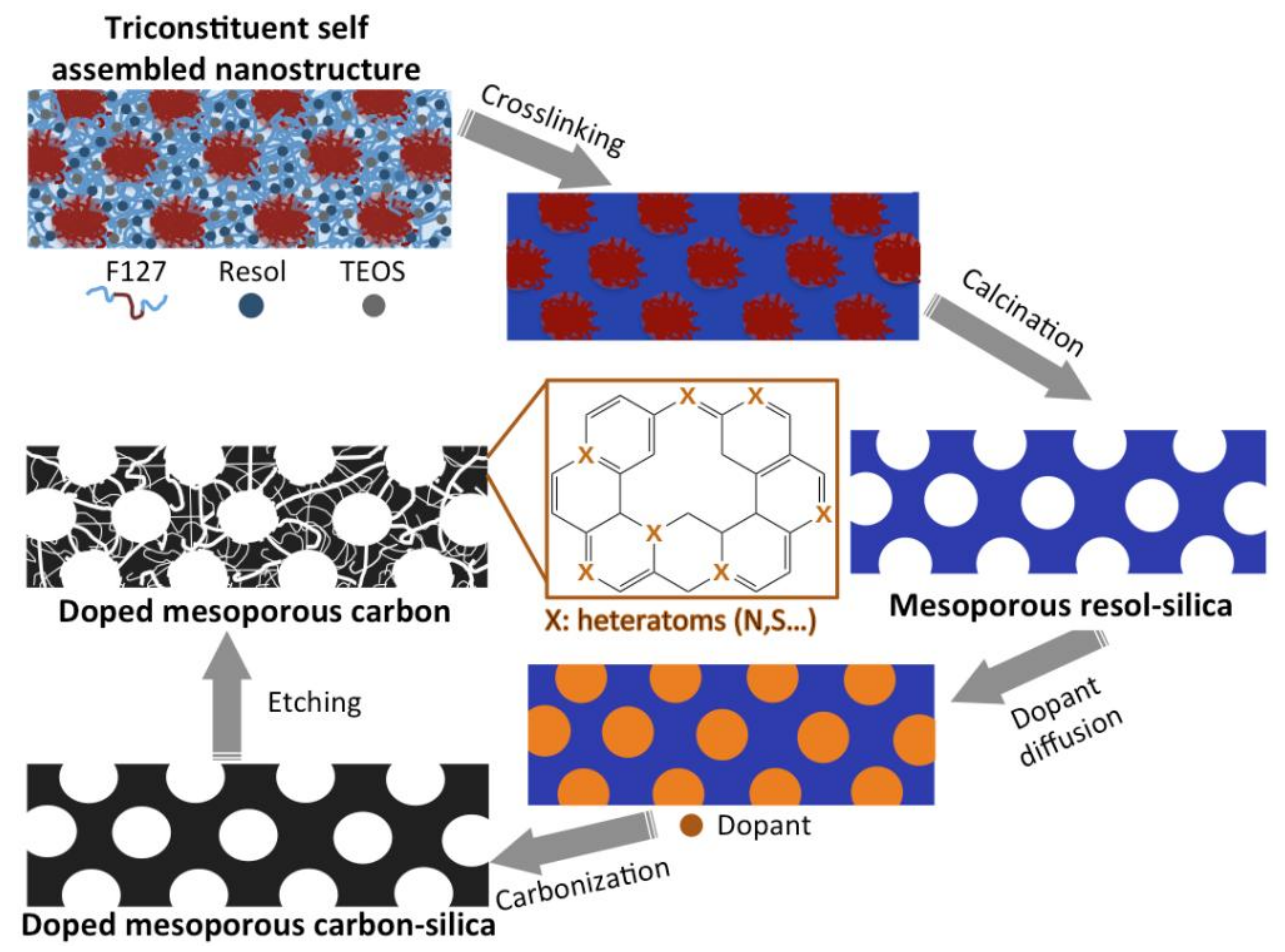

Figure 1. A schematic route of synthesizing the nitrogen, sulfur doped-hierarchical porous carbon (N,S-HPC) 
The N,S-HPC exhibits a Type IV/H3 reversible hysteresis loop (Figure 2a). The ever increasing adsorption from $p / p_{0}=0.8-1$ indicates the presence of macropores due to the defects formed during the doping reactions [36, 37]. The pore size distribution of N,S-HPC (Figure 2b) confirms the co-existence of the micropores (from removal of silica) and mesopores (from F127) in the N,S-HPC. The mesopore size of N,S-HPC $(4.2 \mathrm{~nm})$ is smaller than the ordered mesoporous carbon (OMC, no heteroatom doping, $5.8 \mathrm{~nm}$, Figure S1, Supporting Information, SI), indicating that the expansion of the framework by nitrogen and sulfur during carbonization decreases the mesopore size. Small angle X-ray scattering (SAXS) (Figure S2, SI) confirms this expansion of carbon framework with an increase in the domain-spacing of N,S-HPC $\left(d_{0}=10.4 \mathrm{~nm}\right)$ compared to OMC $\left(d_{0}=9.8 \mathrm{~nm}\right)$. The broad peak at $q^{*} \approx 0.06 \mathrm{~nm}$ for the $\mathrm{N}, \mathrm{S}-\mathrm{HPC}$ suggests a weakly ordered nanostructure that is consistent with the transmission electron microscopy (TEM) micrograph (Figure 2c). Notably, the silica (from TEOS) reinforces the nanostructures and prevents the complete structural collapse upon doping reaction that occurs for analogous samples synthesized without TEOS (Figure S3, SI). Figure 2d-f show the homogeneous distribution of nitrogen and sulfur by energy dispersive X-ray spectroscopy (EDX) within the N,S-HPC. EDX line scans (Figure S4, SI) normal to the pore channels show a constant C:N ratio, indicating these heteroatoms are uniformly doped throughout the carbon walls. 

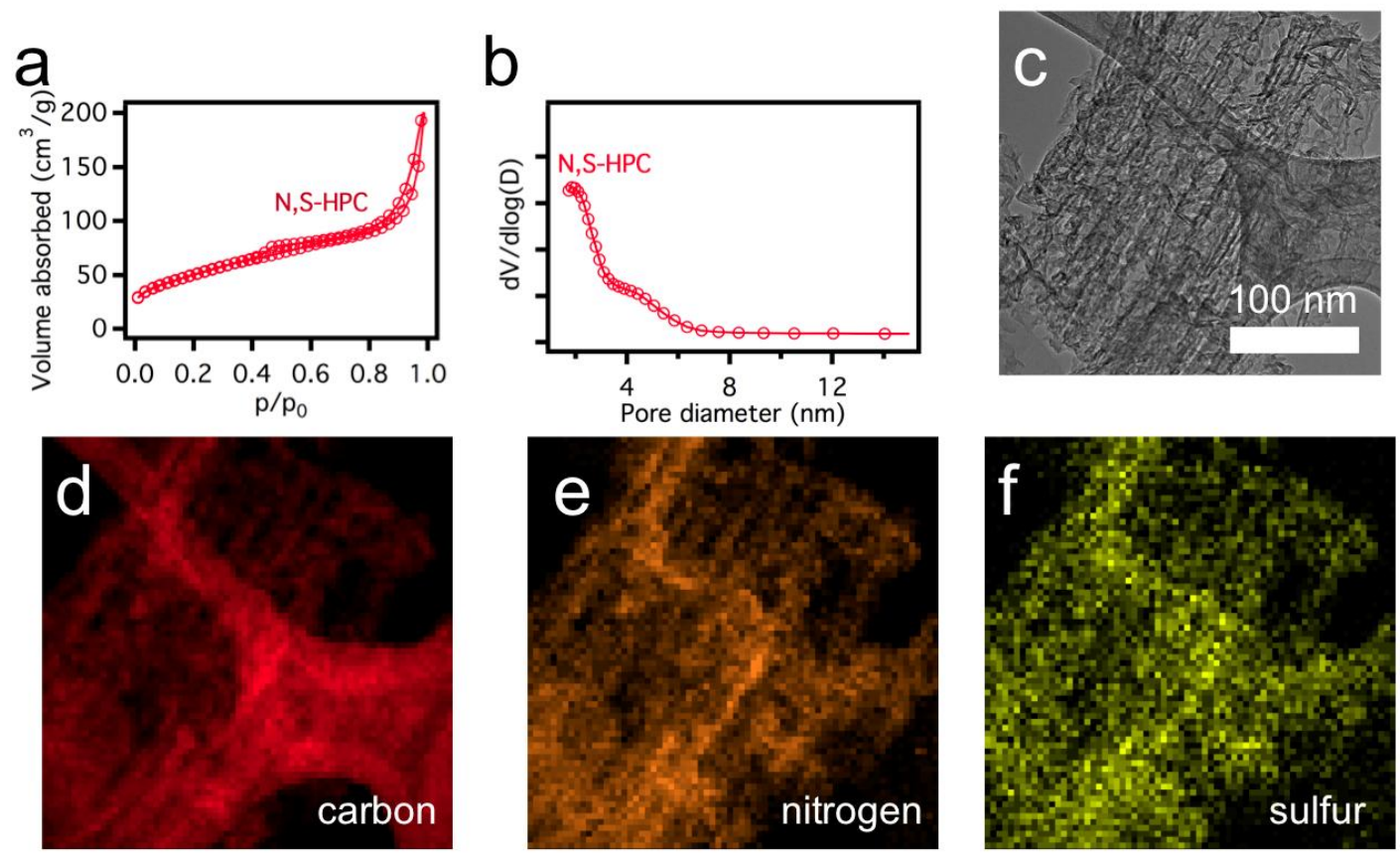

Figure 2. (a) $\mathrm{N}_{2}$ adsorption-desorption isotherm of N,S-HPC and (b) its pore size distribution derived from adsorption isotherm; (c) TEM images of N,S-HPC and its corresponding EDAX elemental mapping of (d) carbon, (e) nitrogen and (f) sulfur.

The simultaneous incorporation of nitrogen and sulfur atoms into the carbon framework is also confirmed by Fourier transform infrared spectroscopy (FTIR) (Figure S5, SI) and X-ray photoelectron spectroscopy (XPS) (Figure S6, SI). A variety of bonding environments for the nitrogen atoms are identified by FTIR including $\mathrm{C}=\mathrm{N}$ stretch, $\mathrm{C}-\mathrm{N}$ stretch, and $\mathrm{C}-\mathrm{N}$ heterocycle (carbon nitride). XPS spectra of the N,S-HPC (Figure S6, SI) indicate the presence of 39.1 atom $\% \mathrm{~N}, 0.9$ atom $\% \mathrm{~S}$ and 6.5 atom $\% \mathrm{O}$ in the carbon framework. During the carbonization step, the dopants can be drawn into the mesopore channels of the polymer matrix by capillary forces on heating. Additionally, the confinement of the nanopores should decrease the effective vapor pressure (capillary concentration) of the liquid melamine $\left(T_{m}=345^{\circ} \mathrm{C}\right)$ and benzyl disulfide $\left(T_{m}=62{ }^{\circ} \mathrm{C}\right)$ to promote their local availability for the doping reaction. This method differs from the standard melt infusion for carbon doping in that the dopant precursors are mixed with the crosslinked carbon-precursor polymer here, rather than with a carbonized material [38, 39]. This 
strategy using the mesoporous resol leads to significantly higher doping content than the standard melt infusion methods as the heteroatoms are dynamically integrated during the carbonization. For co-doping as used here, the difference in the incorporated amount of nitrogen and sulfur in the carbon framework is due to their relative reactivity with carbon [40]. Figure 3ac shows the deconvolution of the C1s, N1s and S2p photoelectron envelopes. For C1s, the binding energies of $284.8 \mathrm{eV}, 285.5 \mathrm{eV}, 287.3 \mathrm{eV}$ and $289.2 \mathrm{eV}$ correspond to the $\mathrm{C}-\mathrm{C}$ (47.1 atom\%), $\mathrm{C}-\mathrm{N}(28.2$ atom\%), $\mathrm{C}=\mathrm{O}(16.7$ atom\%) and $\mathrm{O}-\mathrm{C}=\mathrm{O}$ species $(3.7 \mathrm{atom} \%)$. For N1s, the binding energies of $398.1 \mathrm{eV}, 399.7 \mathrm{eV}$ and $401.0 \mathrm{eV}$ correspond to pyridinic-N (41.3 atom\%), pyrrolic-N (51.9 atom\%) and graphitic-N (6.8 atom\%), respectively. The pyridinic-N and pyrrolic-N could increase pseudo-capacitance, while graphitic-N enhances the conductivity. For $\mathrm{S} 2 \mathrm{p}$, the binding energies at $163.5 \mathrm{eV}, 164.3 \mathrm{eV}$ and $164.9 \mathrm{eV}$ correspond to the $\mathrm{S} 2 \mathrm{p} 3 / 2$ bonds (58.6 atom\%), S-O bonds (5.1 atom\%) and S2p 1/2 species (36.3 atom\%), respectively [24]. These functional groups can improve the affinity and binding energy of the nonpolar carbon atoms with polar polysulfides and thus potentially enhance the cycling stability of metal-S batteries through inhibition of shuttling in electrolytes with high solubility [41, 42] or potentially inhibiting the reactivity with carbonates $[18,31]$. Moreover, the presence of oxygen functional groups in the carbon framework could also promote stablization of sulfur components during cycling $[43,44]$. 

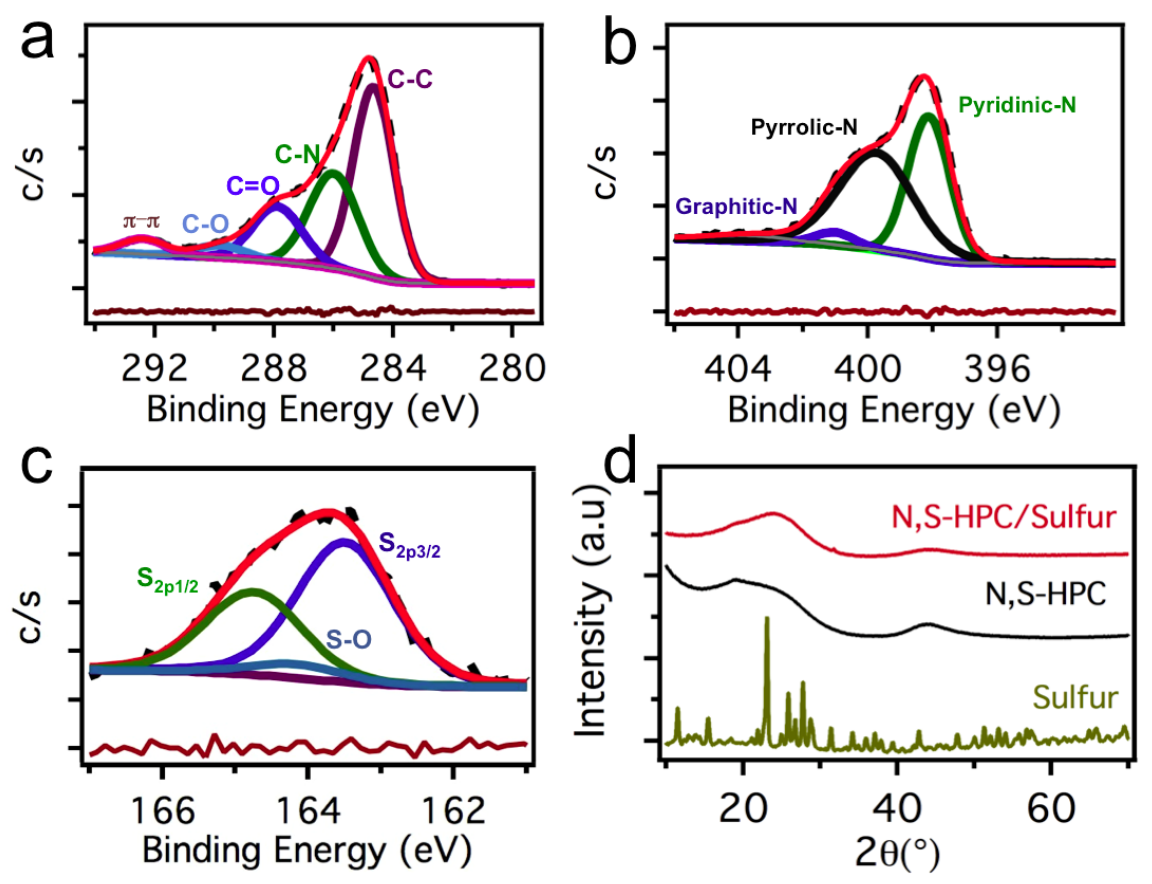

Figure 3. High resolution XPS spectra of (a) C1s, (b) N1s and (c) S2p of N,S-HPC and (d) X-ray diffraction of sublimed sulfur, N,S-HPC and N,S-HPC/sulfur composite with the raw data shown by the black dashed line and the red curve corresponding to the best fit.

The sulfur/N,S-HPC composite in the cathode was prepared by melt diffusion of sulfur into the doped carbon using previously reported conditions for preparation of sulfur/OMC that was used as a cathode in Li-S battery [45]. Thermal treatment of composites at $200{ }^{\circ} \mathrm{C}$ under vacuum for $12 \mathrm{~h}$ is used to remove the excess sulfur to confine the active sulfur ( $22 \mathrm{wt} \% \mathrm{~S}$ in the cathode with the remainder being N,S-HPC/S, carbon black and the binder) to the nanopores (Figure S7, SI). The blocking of the pores by sulfur is confirmed by the adsorption-desorption isotherms of N,S-HPC/S composite with its lack of a hysteresis loop and low total adsorption (Figure S8, SI). As shown in Figure 3d, XRD from the neat sublimed sulfur exhibits multiple sharp diffraction peaks associated with its crystalline structure of $\mathrm{S}_{8}$. For the N,S-HPC, the two broad peaks at $2 \theta$ $=24^{\circ}$ and $43^{\circ}$ in the XRD profile correspond to the graphitic crystallite planes (002) and (100) for turbostratic carbon [46]. However, the diffraction patterns for the N,S-HPC/S composite is 
nearly indistinguishable from the pure N,S-HPC, which suggests that the embedded sulfur in the N,S-HPC is not crystalline and exists as a smaller molecule form than $\mathrm{S}_{8}$. This lack of crystallinity is attributed to the strong polar interaction between sulfur and the doped carbon surface. $\mathrm{S}$ incorporated in PAN also appears to be non-crystalline upon carbonization [18, 31], where the PAN product is N-doped carbon. In contrast, crystalline sulfur is evident from the XRD pattern of OMC/S composites (Figure S9, SI), where these interactions are absent.

To evaluate the effects of the high doping concentration on the performance in a Na-S battery, coin cells consisting of N,S-HPC/S composites as cathode and sodium foil as anode were tested using $\mathrm{NaClO}_{4}$ in $\mathrm{EC} / \mathrm{PC}$. An analogous system using $\mathrm{OMC} / \mathrm{S}$ as the cathode was used as the control. Figure 4a shows the significant difference in the galvanostatic charge/discharge profiles for these two materials. The $\mathrm{OMC} / \mathrm{S}$ shows a voltage plateau at $\mathrm{E}=1.90 \mathrm{~V}$ in the initial discharge with capacity around $381 \mathrm{mAh} / \mathrm{g}$, but fades rapidly to approximately $20 \mathrm{mAh} / \mathrm{g}$ in the second cycle alone. This large decrease in capacity is indicative of loss of active materials (sulfur) due to reaction of sodium polysulfides with carbonates as reported previously for a Na-S battery system [18]. The reaction mechanism of carbonates with polysulfides appears proceed through nucleophilic attack to generate chemistries consistent with thioether and sulfonium functionalities [29]. Generally, the polysulfides in metal-sulfur batteries suffer either from shuttling associated with their high solubility or reactivity with the electrolyte, both of which limits the long term stability of the battery performance. The solubility of polysulfides is very limited in carbonate electrolytes and the active sulfur loss is usually dominated by the chemical reaction of carbonates with polysulfides [47]. Thus, it is likely that this significant capacity loss during the $2^{\text {nd }}$ cycle for Na-S cell with undoped carbon as sulfur host is from this reaction. It 
should be noted that inclusion of low S loadings within mesoporous carbons has been shown to enable decent performance in Li-S batteries when using carbonate electrolytes [48, 49], so the details of the pores and the interaction with $\mathrm{S}$ appears to be critical for determining the reactivity of carbonate electrolytes with polysulfides.

In stark contrast to the undoped carbon/S, the N,S-HPC/S delivers an initial capacity of 450 $\mathrm{mAh} / \mathrm{g}$ with a voltage plateau at $1.4 \mathrm{~V}$ associated with the cleavage of S-S bonds in the composite [31]. In the subsequent discharge cycles, a reversible capacity of $380 \mathrm{mAh} / \mathrm{g}$ is obtained; the voltage profile consists of two regions: (1) a sloping region $(2.7 \mathrm{~V}-1.8 \mathrm{~V})$ from liquid-liquid reaction between $\mathrm{Na}_{2} \mathrm{~S}_{8}$ and $\mathrm{Na}_{2} \mathrm{~S}_{4}$ and (2) a second sloping region (1.8 V- $\left.0.8 \mathrm{~V}\right)$ corresponding to the formation of $\mathrm{Na}_{2} \mathrm{~S}_{2}$ from $\mathrm{Na}_{2} \mathrm{~S}_{4}$. This slope change at $\sim 1.8 \mathrm{~V}$ in the discharge profile is similar to a prior report as characteristics of metal-sulfur chemistry [50]. However, a plateau in voltage around $2 \mathrm{~V}$, corresponding to the reaction between $\mathrm{Na}^{+}$and elemental sulfur is missing. This implies that the N,S-HPC/S composite inhibits the formation of elemental sulfur even when the cell is fully charged $[7,19]$. This absence of a voltage plateau $(E=2 \mathrm{~V})$ for the N,S-HPC/S composite in Figure 4a is similar to several examples for Li-S battery systems, where physical confinement in micropores allows only small sulfur molecules $\left(S_{2}\right.$ or $\left.S_{4}\right)$ to be formed $[31,51,52]$. The N,S-HPC/S discharge curve lacks a second plateau region, suggesting the absence of the transformation from $\mathrm{Na}_{2} \mathrm{~S}_{2}$ to $\mathrm{Na}_{2} \mathrm{~S}$. This is likely associated with the discharge product not commonly being $\mathrm{Na}_{2} \mathrm{~S}$ in $\mathrm{Na}-\mathrm{S}$ cell (the analog of the product in Li-S cells) [9]. Similarly, for an ideal metal-S cell, the CV curve should exhibit two reduction peaks and one oxidation peak, which can be assigned to the formation of $\mathrm{Na}_{2} \mathrm{~S}_{8}$ from $\mathrm{S}_{8}$; formation of $\mathrm{Na}_{2} \mathrm{~S}$ and conversion of sodium polysulfides sulfides to elemental sulfur (2 V - 2.5 V) respectively [19]. 
The absence of $\mathrm{Na}_{2} \mathrm{~S}$ and elemental sulfur upon discharging and charging, respectively, leads to the lack of cathodic and anodic peaks in the cyclic voltammetry (CV) curve as shown in the Figure S10. Thus, based on these observations, the theoretical capacity of the Na-S cell using $\mathrm{N}, \mathrm{S}-\mathrm{HPC} / \mathrm{S}$ is likely $628 \mathrm{mAh} / \mathrm{g}$ considering final charge product as $\mathrm{Na}_{2} \mathrm{~S}_{8}$ and final discharge products as $\mathrm{Na}_{2} \mathrm{~S}_{2}$ as the performance of these cells at low rates exceed the theoretical capacity if the discharge product is $\mathrm{Na}_{2} \mathrm{~S}_{3}(349 \mathrm{mAh} / \mathrm{g})$. After 5 cycles (Figure 4a), both the charge/discharge profiles and CV (Figure S10, SI) are almost identical to the second cycle with nearly no decay in capacitance, indicating excellent cycle stability of the Na-S cell. Comparison between the discharge profiles of undoped carbon and doped carbon as sulfur host illustrates the efficacy of the heteroatom doping on inhibiting the reactions of the polysulfides with carbonate electrolytes. The poor cycling performance of undoped porous carbon hosts for room temperature Na-S batteries is consistent with other literature reports [7], where the poor cycling can be attributed to either polysulfide shuttling or side reactions with the electrolytes.

Figure $4 \mathrm{~b}$ illustrates the rate performance of Na-S battery using N,S-HPC/S as the cathode. At lowest current rate $(115 \mathrm{~mA} / \mathrm{g})$, the capacity is around $455 \mathrm{mAh} / \mathrm{g}$, and decreases to approximately $380 \mathrm{mAh} / \mathrm{g}, 345 \mathrm{mAh} / \mathrm{g}, 285 \mathrm{mAh} / \mathrm{g}$ and $196 \mathrm{mAh} / \mathrm{g}$ after sequentially increasing the current rate to $230 \mathrm{~mA} / \mathrm{g}, 575 \mathrm{~mA} / \mathrm{g}, 1150 \mathrm{~mA} / \mathrm{g}$ to $2300 \mathrm{~mA} / \mathrm{g}$. The high capacity (443 $\mathrm{mAh} / \mathrm{g}$ ) is mostly recovered on decreasing the current rate back to $115 \mathrm{~mA} / \mathrm{g}$. The ability to use non-conductive $\mathrm{S}$ as the active material at such high rates for this cell is attributed to the structural and chemical nature of the N,S-HPC, which provides high contact area between nonconductive sulfur and carbon to facilitate electron transfer and the nitrogen functionality of carbon framework that improves the electrolyte wettability. It should be noted that sodium 
batteries based on $\mathrm{FeS}_{2}$ exhibit high rate performance when a similar potential window is selected to prevent formation of $\mathrm{Na}_{2} \mathrm{~S}$ and crystalline phase transitions [53]. Similarly, the $\mathrm{S}$ in the N,S-HPC remains amorphous throughout the charge-discharge, which may facilitate the capacity performance and reversibility at high rates. The limited fade in the capacity of Na-S battery is confirmed by the extended cycling at $230 \mathrm{~mA} / \mathrm{g}$ (Figure $4 \mathrm{c}$ ). The capacity remains at $378 \mathrm{mAh} / \mathrm{g}$ after 350 cycles, $\approx 2 \%$ fade in capacity, which is significantly less than prior reports for room temperature Na-S batteries [19]. The energy density is approximately $150 \mathrm{Wh} / \mathrm{kg}$ (mass basis of the total cathode) for this N,S-HPC/S cathode. This energy density compares favorably to current stationary energy storage and meets near-term future requirements [54].

The excellent cycle stability is important for potential stationary energy storage applications. Here this stability can be attributed to the high concentration of nitrogen functional group in the carbon framework. The inclusion of heteroatoms into the carbon framework could modify the polarity of pore surface and thus increasing the binding energy with polysulfides and promoting chemical adsorption of sulfur [55]. This enhanced affinity could trap polar sodium polysulfides in the pores during the cycling to prevent reactions with the carbonate electrolyte [56]. One additional interaction to consider in the cycling stability is that of Na-N. The formation of metal$\mathrm{N}$ bond has been previously reported for improving the cycle stability of lithium-sulfur battery when using polyaniline nanotubes or carbon nitride as the sulfur host $[52,57]$. With the high pyrrolic-nitrogen content in the N,S-HPC, these nitrogen can bond with $\mathrm{Na}^{+}$via a lone pair electron through a favorable Na-N interaction, leading to a coordination-like interaction [58]. High-resolution XPS (Figure S11, SI) can identify the Na-N from the N1s spectrum of the electrode after discharge. The N1s spectrum for the discharged N,S-HPC shows an asymmetric 
broadening towards lower binding energy in comparison to the virgin N,S-HPC. This difference is indicative of a change in chemical environment for the $\mathrm{N}$ in the $\mathrm{N}, \mathrm{S}-\mathrm{HPC}$ as there is no $\mathrm{N}$ in the electrolyte. A good fit of this N1s spectrum for the discharged material can be obtained with the addition of a new peak at $397.2 \mathrm{eV}$ that was not present in the virgin N,S-HPC (Figure 3b) and this binding energy is consistent with prior reports for Na-N [58]. The XPS spectra provides evidence of a Na-N interaction between doped porous carbon and the sodium $\mathrm{Na}-\mathrm{S}$ - species. Thus, the end groups in sodium sulfides $\left(\mathrm{Na}-\mathrm{S}-\mathrm{S}_{\mathrm{n}-2}-\mathrm{S}-\mathrm{Na}\right)$ provides an additional mechanism for N,S-HPC to effectively trap sodium polysulfides during cycling, which appears to prevent reactions with the carbonate electrolyte. In addition to the heteroatom doping of the carbon, several other factors may be important in the cycle stability. Prior work has demonstrated that preventing formation of $\mathrm{Na}_{2} \mathrm{~S}$ can improve the lifetime for sodium batteries based on $\mathrm{FeS}_{2}$, which was based on the selection of the cut-off potential $(0.8 \mathrm{~V})$ [53]. The potential window for this high performance $\mathrm{FeS}_{2}$ electrodes [53] closely matches the potential used here and $\mathrm{Na}_{2} \mathrm{~S}$ is not formed on discharge, which may be important for the stability of Na-S batteries. Similarly, the single phase (amorphous) of the sulfur/sulfides present through the charge-discharge process may also facilitate the reversibility.

A simple H-shape diffusion model (Figure S12, SI) was used to confirm that the heteroatoms in the N,S-HPC are able to effectively trap sodium polysulfides even for an electrolyte with high sodium polysulfide solubility (TEGDME). The diffusion of $\mathrm{Na}_{2} \mathrm{~S}_{8}$ across the junction containing either OMC or N,S-HPC coated separator is driven by a concentration gradient. This diffusion process was significantly mitigated by the N,S-HPC coated separator, while the sodium sulfides diffuse readily across with the $\mathrm{OMC}$ coated separator in $30 \mathrm{~min}$. The strong binding (Na-N) also 
helps to explain the charge product of $\mathrm{Na}_{2} \mathrm{~S}_{8}$ rather than $\mathrm{S}_{8}$ [31]. The doping in the N,S-HPC contributes little to the capacity as shown in Figure S13 with energy density almost exclusively due to the sulfur confined within the N,S-HPC. This result further illustrates that sulfur is the major active material. This can be also demonstrated from discharge/charge profiles (Figure S14), where this Na-S cell is able to deliver a capacity of more than $250 \mathrm{mAh} / \mathrm{g}$ in the potential window of $2.7 \mathrm{~V}-1.0 \mathrm{~V}$, which significantly exceeds the performance for Na-ion batteries with highly-doped carbon $(>10 \mathrm{at} \%)$ as electrodes in this potential window $(<40 \mathrm{mAh} / \mathrm{g})$ [59].

In order to further investigate the product when completely charged and discharged, electrochemical impedance spectroscopy (EIS) was carried out at the fully charged and discharged states during the first 10 cycles. Only one semicircle in the high-frequency (HF) region reflecting the charge transfer process at the conductive matrix interface is observed in all cases (Figure S15, SI). As shown in Figure 4d, the resistance of the fully charged battery $(\approx 230$ $\Omega)$ is lower than the fully discharged $(\approx 395 \Omega)$, which is contrary to previous reports regarding with metal-sulfur battery [60]. However, this phenomenon correlates well with our observations for the fully charged product of $\mathrm{Na}_{2} \mathrm{~S}_{8}$ instead of pure insulating elemental sulfur. Moreover, long chain polysulfides exhibit higher ionic conductivity than their shorter counterparts [61]. The variation in resistance after each galvanostatic charge/discharge protocol is coherent in the first 10 cycles, further confirming the excellent stability during cycling of this Na-S battery.

The ultra-long term cycle stability of the Na-S cell was examined at a high current density of 4.6 A/g as shown in Figure 4d. After the initial discharge with only 55.4\% Coulombic efficiency for the first cycle, the capacity is approximately $128 \mathrm{mAh} / \mathrm{g}$ with only $3 \%$ fade in capacity after 
8000 cycles with $>99.5 \%$ Coulombic efficiency. After 10,000 cycles, the discharge capacity is still greater than $84 \mathrm{mAh} / \mathrm{g}$. It is important to note that even at high current density of $4.6 \mathrm{~A} / \mathrm{g}$, the capacity at potential window of $2.7 \mathrm{~V}-1 \mathrm{~V}$ is still over $70 \%$ of total discharge capacity, which distinct from other Na-ion cells with doped carbon as electrodes. (Figure S16, SI) The N,SHPC/S cathode provides many advantages including excellent cycle stability (> 8000 cycles) and a fast charging time of around $3 \mathrm{~min}$ at current density of $4.6 \mathrm{~A} / \mathrm{g}$ with a power density of 9200 $\mathrm{W} / \mathrm{kg}$ cathode, in the range of high power lithium ion batteries [62]. This high power density of Na-S cell can be attributed to the hierarchical porous structure of N,S-HPC. In the N,S-HPC/S composite, the sulfur is confined within the nanopores of the doped carbon, where the size of the pores dictates that a conductive material (carbon) is within $2 \mathrm{~nm}$ of any non-conductive sulfur. This provides electrical contact to the $\mathrm{S}$ to allow this fast charging-discharging rate. The apparent oscillations in the discharge capacity at both slow (Figure 4c) and fast (Figure 4d) rates track well with the heating-cooling duty cycle for temperature control in the room where the battery was tested (Figure S17, SI). The capacity of the N,S-HPC/S is sensitive to the local temperature where increasing temperature leads to an enhanced mobility of sodium ions during cycling at high current density $(4.6 \mathrm{~A} / \mathrm{g})$. As shown in Figure S17, the discharge capacity of Na-S cell increases approximately $20 \%$ when the operating temperature increases from $24^{\circ} \mathrm{C}$ to $32^{\circ} \mathrm{C}$ and then reversibly decreases when the temperature is decreased back to $24^{\circ} \mathrm{C}$. This temperature dependence of the capacity is consistent with the oscillatory variation in the capacities in Figures $4 \mathrm{c}$ and $4 \mathrm{~d}$. These changes do not impact the long-term stability of the battery, so the inhibition of reactions of the sodium sulfide with the electrolyte by the N,S-HPC is not impacted by these shifts in temperature. At the same current density, an analogous sample of N-HPC (34 atom\% nitrogen without any S doping)/sulfur composite exhibits stable cycle performance over 1000 
cycles with a slightly lower capacity $(112 \mathrm{mAh} / \mathrm{g})$ compared to the N,S-HPC/S, suggesting that the inhibition of polysulfide reactivity with the carbonate electrolyte is mainly due to high doping concentration of nitrogen atoms, while sulfur atoms in the carbon framework could contribute to the pseudo-capacitance (Figure S18, SI). The improved cycle stability in the N,SHPC/S composite compared to prior work is attributed to: First, the stable carbon wall confines the volumetric expansion of sulfur during the transformation to sodium sulfides; second, the removal of excess sulfur prevents the loss of active materials during cycling; third, introduction of heteroatoms including nitrogen and sulfur into the carbon matrix changes the electronic band of carbon framework that immobilizes the polysulfide, and fourth, the strong sodium-nitride interaction during discharge further confines the sodium sulfides to the pores and thus the reaction of polysulfide with electrolytes is almost completely inhibited.

To verify this near complete inhibition of S loss through shuttling and side reactions, the Na-S battery was disassembled after 1000 cycles. As displayed in the inset of Figure S19, traces of yellow polysulfides are clearly observed in the glass fiber separator using $\mathrm{OMC} / \mathrm{S}$ as the cathode and this transport of polysulfides to the separator is prevented when using N,S-HPC as the sulfur host. [50] The UV-vis spectra (Figure S19, SI) of the electrolyte after 1000 cycles confirms the suppression of dissolution of polysulfides by N,S-HPC with nearly identical spectra for the electrolyte reference and the electrolyte after 1000 cycles. A broad peak at $550 \mathrm{~nm}$ appears when using $\mathrm{OMC} / \mathrm{S}$ as the cathode, which corresponds to the yellow color of sodium polysulfides. 


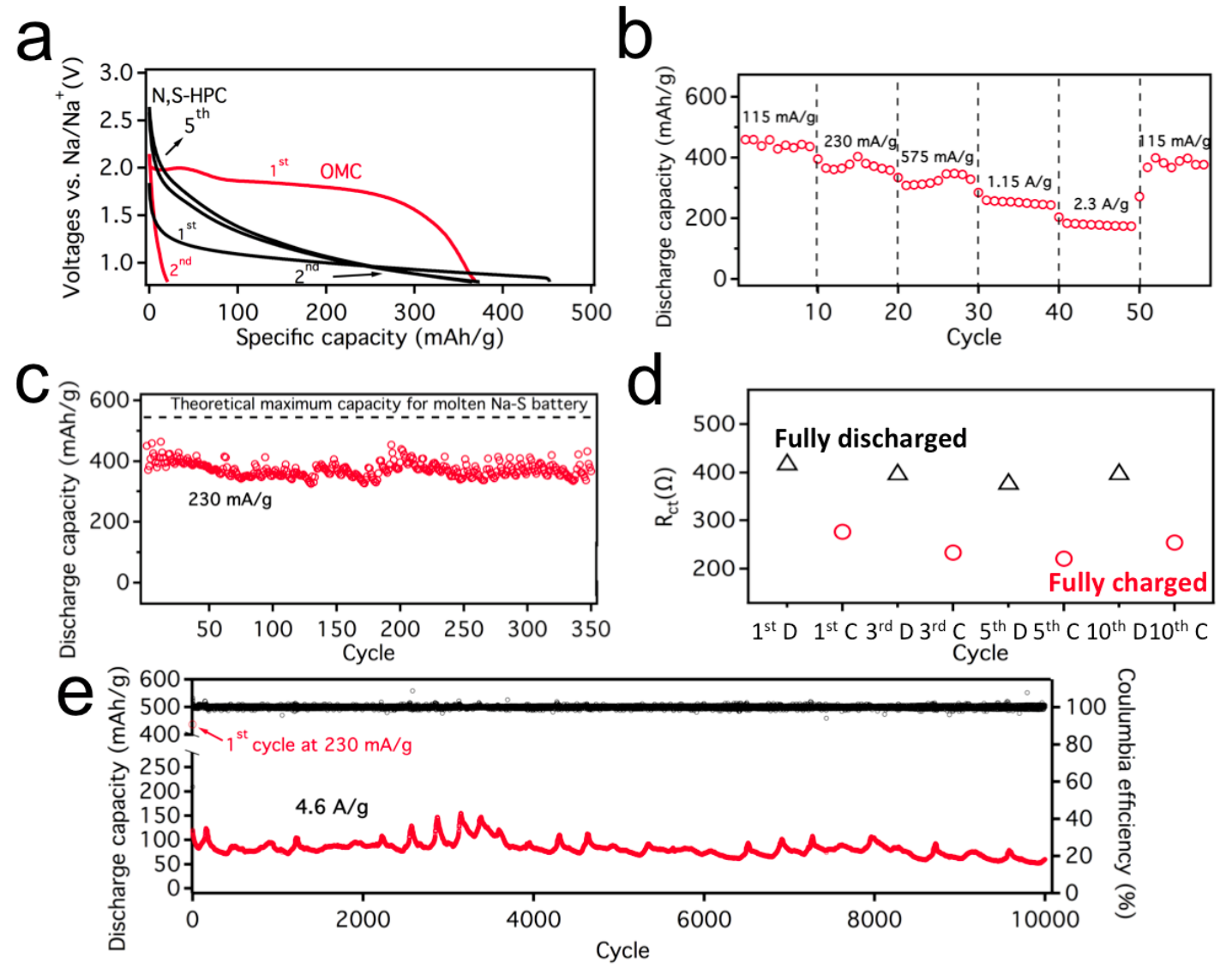

Figure 4. (A) Electrochemical discharge curves of N,S-HPC/S of the first two and fifth cycle and $\mathrm{OMC} / \mathrm{S}$ during the first two cycles; (B) rate performance of N,S-HPC/S at various current density; (C) the discharge capacity of N,S-HPC/S at $230 \mathrm{~mA} / \mathrm{g}$; (D) the resistance of charged and discharged Na-S cell with N,S-HPC/S as electrodes after $1^{\text {th }}, 3^{\text {rd }}, 5^{\text {th }}$ and $10^{\text {th }}$ cycle and $(\mathrm{E})$ the discharge capacity of N,S-HPC/S at $4.6 \mathrm{~A} / \mathrm{g}$ cycled over ten thousand times.

\section{Conclusions}

In conclusion, we report a facile method of synthesizing hierarchical porous carbon with high heteroatoms concentration ( 40 atom $\%)$ for room temperature sodium-sulfur battery. The large concentration of heteroatoms in the carbon framework prevents the reaction of polysulfides with the carbonate electrolyte. Similarly, the transport of polysulfides is inhibited in these highly doped porous carbons when using TEGME. The cells fabricated with the N,S-HPC/S cathodes exhibit ultra-long cycle life over 10,000 cycles with less than $20 \%$ decay in capacity (only $3 \%$ 
after 8000 cycles) at a high current density $(4.6 \mathrm{~A} / \mathrm{g})$ in these coin cells. Larger battery cells are required to determine if this very long cycle life for these $\mathrm{N}, \mathrm{S}-\mathrm{HPC} / \mathrm{S}$ cathodes is scalable. This high doping strategy for porous carbons is a promising strategy to enable sufficient performance for room temperature sodium-sulfur batteries in stationary, grid-level energy storage.

\section{Acknowledgement}

This work was supported by the National Science Foundation under [Grant no. 1159295] and [Grant CBET-1336057]. The authors thank Nikolov Zhorro for the XPS measurement and thank Min Gao for the help in TEM measurement. $\ddagger$ Z. Q and Y. C contributed equally.

\section{References}

[1] Z. Yang, J. Zhang, M. C. W. Kintner-Meyer, X. Lu, D. Choi, J. P. Lemmon, J. Liu, Chem. Rev. 111 (2011) 3577-3613

[2] D. Larcher, J. M. Tarascon, Nat. Chem. 7 (2015) 19-29.

[3] B. Dunn, H. Kamath, J.-M. Tarascon, Science. 334 (2011) 928-935.

[4] G. L. Soloveichik, Chem. Rev. 115 (2015) 11533-11558.

[5] H. Chen, T. N. Cong, W. Yang, C. Tan, Y. Li, Y. Ding, Prog. Nat. Sci. 19 (2009) 291-312.

[6] K. B. Hueso, M. Armand, T. Rojo, Energy Environ. Sci. 6 (2013) 734-749.

[7] H. Ryu, T. Kim, K. Kim, J.-H. Ahn, T. Nam, G. Wang, H.-J. Ahn, J. Power Sources 196 (2011) 5186-5190. 
[8] S. Wei, S. Xu, A. Agrawral, S. Choundhury, Y. Lu, Z. Tu, L. Ma, L. A. Archer, Nature Comm 7 (2016) 11722

[9] J. Wang, J. Yang, Y. Nuli, R. Holze, Electrochem. Comm. 9 (2007) 31-34.

[10] X. Yu, A. Manthiram, J. Phys. Chem. C. 118 (2014) 22952-22959.

[11] C.-W. Park, H.-S. Ryu, K.-W. Kim, J.-H. Ahn, J.-Y. Lee, H.-J. Ahn, J. Power Sources 165 (2007) 450-454.

[12] S. Wenzel, H. Metelmann, C. Raiss, A. K. Duerr, J. Janek, P. Adelhelm, J. Power Sources 243 (2013) 758-765.

[13] I. Bauer, M. Kohl, H. Althues, S. Kaskel, Chem. Comm. 50 (2014) 3208-3210.

[14] X. Wang, L. Fan, D. Gong, J. Zhu, Q. Zhang, B. Lu, Adv. Funct. Mater. 26 (2016) 11041111

[15] D. Gong, B. Wang, J. Zhu, R. Podila, A. M. Rao, X. Yu, Z. Xu, B. Lu, Adv. Energy Mater DOI: $10.1002 /$ aenm/201601885

[16] X. Yu, A. Manthiram, Adv. Energy Mater. 5 (2015) 1500350.

[17] X. Yu, A. Manthiram, Chem. Eur. J. 21 (2015) 4233-4237.

[18] T. H. Hwang, D. S. Jung, J.-S. Kim, B. G. Kim, J. W. Choi, Nano Lett. 13 (2013) 45324538.

[19] S. Xin, Y.-X. Yin, Y.-G. Guo, L.-J. Wan, Adv. Mater. 26 (2014) 1261-1265.

[20] Y. Chen, W. Liang, S. Li, F. Zou, S. Bhaway, Z. Qiang, M. Gao, B. D. Vogt, Y. Zhu, J. Mater. Chem. A, 4 (2016), 12471-12478

[21] X. Yu, A. Manthiram, Chemelectrochem 1 (2014) 1275-1280. 
[22] X. Yu, A. Manthiram, J. Phys. Chem. Lett. 5 (2014) 1943-1947.

[23] A. Manthiram, X. Yu, Small. 11 (2015) 2108-2114

[24] M. Kohl, F. Borrmann, H. Althues, S. Kaskel, Adv. Energy Mater. 6 (2016) 1502185

[25] L. Fan, R. Ma, Y. Yang, S. Chen, B. Lu, Nano Energy 28 (2016) 304-310.

[26] G. Zhou, E. Paek, G. S. Hwang, A. Manthiram, Nat. Commun. 6 (2015) 7760

[27] Q. Pang, J. Tang, H. Huang, X. Liang, C. Hart, K. C. Tam, L. F. Nazar, Adv. Mater. 27 (2015) 6021-6028.

[28] J. Song, Z. Yu, M. L. Gordin, D. Wang, Nano Lett. 16 (2016) 864-870.

[29] J. Gao, M. A. Lowe, Y. Kiya, H. D. Abruna, J. Phys. Chem. C 115 (2011) 25132-25137.

[30] I. Kim, J.-Y. Park, C. Park, J.-W. Park, J.-P. Ahn, J.-H. Ahn, K.-W. Kim, H.-J. Ahn. J. Electrochem. Soc. 163 (2016) 611-616.

[31] S. Wei, L. Ma, K. E. Hendrickson, Z. Tu, L. A. Archer, J. Am. Chem. Soc. 137 (2015) 12143-12152.

[32] Z-W. She, J. Sun, Y. Sun, Y. Cui, ACS Cent Sci. 1 (2015) 449-455

[33] C. Xue, B. Tu, D. Zhao, Adv. Funct.Mater. 18 (2008) 3914.-3921.

[34] Z. Qiang, Y. Guo, H. Liu, S. Z. D. Cheng, M. Cakmak, K. A. Cavicchi, B. D. Vogt, ACS Appl. Mater. Interfaces. 7 (2015) 4306-4310.

[35] Z. Qiang, B. Gurkan, J. Ma, X. Liu, Y. Guo, M. Cakmak, K. A. Cavicchi, B. D. Vogt, Microporous. Mesopoorous. Mater. 227 (2016) 57-64 
[36] C. Zhang, L. Fu, N. Liu, M. Liu, Y. Wang, Z. Liu, Adv. Mater. 2011, 23, 1020-1024.

[37] W.-h. Lee, J. H. Moon, ACS Appl. Mater. Interfaces. 6 (2014)13968-13976.

[38] S. M. Mahurin, J. S. Lee, X. Wang, S. Dai, J. Membrane Sci. 368 (2011) 41-47.

[39] N. Fechler, N. P. Zussblatt, R. Rothe, R. Schlogl, M. G. Willinger, B. F. Chmelka, M. Antonietti, Adv. Mater. 28 (2016) 1287-1294.

[40] W. Kicinski, M. Szala, M. Bystrzejewski, Carbon 68 (2014) 1-32.

[41] H.-J. Peng, T.-Z. Hou, Q. Zhang, J.-Q. Huang, X.-B. Cheng, M.-Q. Guo, Z. Yuan, L.-Y. He, F. Wei, Adv. Mater. Interfaces 1 (2014) 1400227

[42] K. A. See, Y.-S. Jun, J. A. Gerbec, J. K. Sprafke, F. Wudl, G. D. Stucky, R. Seshadri, ACS Appl. Mater. Interfaces 6 (2014) 10908-10916.

[43] L. Fan, B. Lu, Small, 12 (2016) 2783-2791

[44] C. Luo, Y. Zhu, O. Borodin, T. Gao, X. Fan, Y. Xu, K. Xu, C. Wang, Adv. Funct. Mater 26 (2016) 745-752

[45] X. Ji, K. T. Lee, L. F. Nazar, Nat. Mater. 8 (2009) 500-506.

[46] Z. Q. Li, C. J. Lu, Z. P. Xia, Y. Zhou, Z. Luo, Carbon 45 (2007) 1686-1695.

[47] T. Yim, M-S. Park, J-S. Yu, K. Kim, K. Im, J-H. Kim, G. Jeong, Y. Jo, S-G. Woo, K.

Kang, I. Lee, Y-J. Kim, Electrochim. Acta 107 (2013) 454-460

[48] C. Lai, X. P. Gao, B. Zhang, T.Y. Yan, Z. Zhou, J. Phys. Chem. C 113 (2009) 4712-4716.

[49] B. Zhang, C. Lai, Z. Zhou, X. P. Gao, Electrochim. Acta 54 (2009) 3708-3713. 
[50] G. Li, J. Sun, W. Hou, S. Jiang, Y. Huang, J. Geng, Nature. Comm. 7 (2016) 10601

[51] B. Zhang, X. Qin, G. R. Li, X. P. Gao, Energy Environ. Sci. 3 (2010) 1531-1537.

[52] J. Guo, Z. Yang, Y. Yu, H. D. Abruna, L. A. Archer, J. Am. Chem. Soc. 135 (2013) 763767.

[53] Z. Hu, Z. Zhu, F. Cheng, K. Zhang, J. Wang, C. Chen, J. Chen, Energy Environ. Sci 8 (2015) 1309-1316

[54] W. Wang, Q. Luo, B. Li, X. Wei, L. Li, Z. Yang. Adv. Funct. Mater. 23 (2013) 970-986.

[55] Z. W. She, Y. Sun, Q. Zhang, Y. Cui, Chem. Soc. Rev. 45 (2016) 5605-5634

[56] P. Zhu, J. Song, D. Lv, D. Wang, C. Jaye, D. A. Fischer, T. Wu, Y. Chen J. Phys. Chem. C. 118 (2014) 7765-7771

[57] L. Xiao, Y. Cao, J. Xiao, B. Schwenzer, M. Engelhard, L. V. Saraf, Z. Nie, G. J. Exarhos, J. Liu, Adv. Mater. 24 (2012) 1176-1181

[58] Z. W. Seh, H.Wang, P.-C. Hsu, Q. Zhang, W. Li, G. Zheng, H. Yao, Y. Cui, Energy Environ. Sci. 7 (2014) 672-676.

[59] Z. Wang, L. Qie, L. Yuan, W. Zhang, X. Hu, Y. Huang, Carbon 55 (2013) 328-334

[60] J. Zhou, R. Li, X. Fan, Y. Chen, R. Han, W. Li, J. Zheng, B. Wang, X. Li, Energy Environ. Sci. 7 (2014) 2715-2724.

[61] D. R. Wang, K. H. Wujcik, A. A. Teran, N. P. Balsara, Macromolecules 48 (2015) 48634873. 
[62] X. Dong, L. Chen, J. Liu, S. Haller, Y. Wang, X. You, Sci. Adv. 2 (2016) e1501038. 


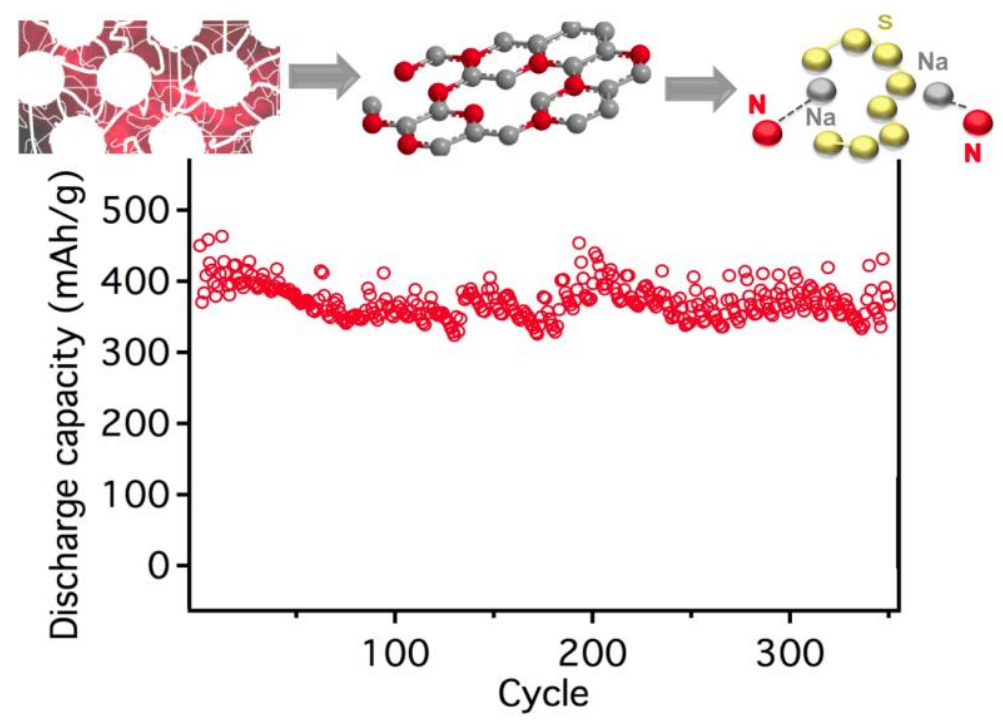

Highly doping $(\approx 40$ at $\%$ ) porous carbons enable stable Na-S battery performance using carbonate-based electrolytes. 